\title{
Análisis didáctico de un tema de diversidad, desarrollado en un libro de texto escolar colombiano
}

\section{Didactic analysis of a diversity subject, developed in a colombian school textbook}

\author{
Por: Alejandra Correa y Diana Villa ${ }^{1}$
}

\section{Resumen}

Se presenta un análisis de textos escolares para un tema de diversidad de un libro de ciencias naturales de grado noveno. El objetivo de este trabajo es contribuir con el mejoramiento de un texto escolar de amplio uso para enriquecer la enseñanza d ela diversidad y de la biología en nuestro país. Las conclusiones del presente trabajo son las siguientes: I) Los contenidos del texto analizado se presentan en foma estructurada, coherente y equilibrada. II) Se detectaron algunos errores que pueden generar confusión en los estudiantes y que deben ser corregidos. III) El interés de los autores en facilitar el aprendizaje, se observa en el uso de una presentación llamativa y atractiva, un lenguaje claro y varios contenidos actitudinales. IV) El espacio para desarrollar los contenidos es bastante limitado, sin embargo, se logra una presentación equilibrada de los mismos. V) Las actividades prácticas propuestas en el texto son fáciles de implementar en las instituciones educativas colombianas e incorporan las experiencias cotidianas de los alumnos.

Palabras claves: texto escolar, diversidad, clasificación, taxonomía, enseñanza de la biología.

\section{Abstract}

A school textbook analysis of a topic in diversity, from a natural sciences book, is presented. The objective of this work is to contribute with the improvement of a widely used school textbook in order to enrich the diversity and biology teaching in our country. The conclusions of this work are the following: I) The

${ }^{1}$ Estudiantes de la Especialización en Enseñanza de la Biología, Universidad Pedagógica Nacional. acorream@gmail.com 
contents of the analyzed texts are presented in a structured, coherent and equilibrated way. II) Some errors that can generate confusion in the students are detected and they must be corrected. III) The author's interest in making learning easier can be observed in the use in a showy and attractive presentation, a clear language and several attitudinal contents. IV) The space for the contents development is pretty limited; however, an equilibrated presentation of them is achieved. V) The practical activities proposed in the text are easy to implement in Colombianeducational institutions and incorporate students' common experiences.

Key words: school textbook, diversity, classification, taxonomy, biology teaching.

\section{Introducción}

Teniendo en cuenta la gran riqueza en diversidad de nuestro territorio y los problemas sociales y políticos que amenazan con su destrucción, es importante evaluar la forma en la cual esta característica vital de nuestro país es incorporada al currículo de Ciencias Naturales y Educación Ambiental. En especial, nos interesa analizar el tratamiento que uno de los libros de texto más usados por los estudiantes colombianos da a este tema tan importante.

La biología define a la biodiversidad como la variedad y variabilidad de los seres vivos y de los ecosistemas que éstos integran. Los componentes de la diversidad biológica se organizan en tres niveles: el de los genes, que constituyen las bases moleculares de la herencia; el de las especies, que son conjuntos de organismos afines capaces de reproducirse entre sí y el de los ecosistemas, que son complejos funcionales formados por los organismos y el medio físico en el que habitan (Crisci, 2001).

De acuerdo con Crisci (2001), las especies se constituyen en la herramienta científica más utilizada para medir la biodiversidad y, como tal, la primera para definir políticas de conservación de la misma. Por ello resulta imprescindible conocer las especies que habitan el planeta y ubicarlas en un marco clasificatorio fundamentado en hipótesis científicas. Esto se ve reflejado en los estándares educativos para el área de Ciencias Naturales y Educación Ambiental propuestos por el Ministerio de Educación Nacional (MEN), en los que se incluye el desarrollo en los alumnos de un conocimiento básico sobre la clasificación de los organismos.

Toda educación proviene de alguna imagen del futuro. Cuando un alumno pregunta para qué tiene que aprender álgebra, no le decimos "porque tu abuelo la aprendía", sino que le decimos porque la necesitará en el futuro. Esto presupone que los padres, los responsables de los programas de estudio y los docentes desarrollan una serie de hipótesis respecto a cómo será la sociedad más adelante. Esto significa preparar a los 
jóvenes a planear la sociedad y sus propias vidas en función de las circunstancias en las que vivirán, o dicho en otras palabras, atribuir significado a lo que se enseña (Crisci, 2001).

Una fuerte hipótesis sobre el futuro incluye al problema de la biodiversidad. Lo anterior se ve reflejado en la declaración del director general de la UNESCO, Koïchiro Matsuura, quien señala que "Se necesita un esfuerzo enorme en el tema educación y biodiversidad con el objeto de crear una conciencia global de los problemas que afrontamos. Sólo una sociedad educada sobre la biodiversidad puede crear las condiciones que nos lleven a un futuro sustentable. La UNESCO está desarrollando junto a otros organismos una nueva iniciativa global en esta dirección, que tendrá como objetivo la educación, el entrenamiento, y el desarrollo de una conciencia pública sobre el tema biodiversidad" (Biology International № 39, julio 2000).

Esta situación exige nuevos objetivos educacionales que contemplen la problemática de la biodiversidad y que resalten el papel que la sistemática biológica tiene en la prevención y minimización del problema (Crisci,1994). Así, de acuerdo con Crisci (1994), los nuevos objetivos educacionales de la sociedad con referencia a la biodiversidad son:

* Crear conciencia sobre la pérdida de la biodiversidad.

* Fundamentar la necesidad de aprender más acerca de la biodiversidad.

* Producir ciudadanos informados y educados sobre la biodiversidad.

Por otra parte, los objetivos educacionales con respecto a la biodiversidad, desde el punto de vista de los estudiantes, son los siguientes según Crisci (1994):

* Aprender a valorar la biodiversidad.

* Aprender a valorar la sistemática.

* Aprender a comunicar información utilizando conceptos sistemáticos.

* Aprender a tomar decisiones racionales con respecto a la biodiversidad.

Un cambio educativo que contemple estas necesidades de la sociedad y de los estudiantes debe partir de los siguientes fundamentos (Crisci, 1994):

* La biodiversidad es un recurso global que necesita ser preservado.

* La sistemática es la herramienta básica para el estudio de la biodiversidad.

* La enseñanza de la sistemática juega un papel importante en la conservación de la biodiversidad.

* El aprendizaje de la sistemática es un proceso activo y constructivo.

* La enseñanza de la sistemática debe estar basada en problemas reales, que demuestren el significado de la biodiversidad para la sociedad y las personas.

Como una pequeña contribución a la mejora de la enseñanza de la diversidad en nuestro país, se desarrolla en este artículo un análisis sobre la forma en que este tema es presentado en un libro de texto de ciencias naturales colombiano. Con respecto a este tipo de material didáctico, es importante señalar que los textos escolares no son un medio o material neutro respecto a posiciones ideológicas, sino que ejercen una notable 
influencia en la configuración de la cultura escolar, en la implementación curricular y en las prácticas docentes del profesorado. El formato actual de los libros de texto consiste en un contenido conceptual y en un repertorio de ejemplos, actividades y problemas, generalmente acompañados con algunas biografía de científicos y lecturas complementarias (Zapata, 2006).

Según la tesis de Zulma Zapata (2006), titulada: Evaluación de contenidos y actividades de enseñanza de la Microbiología en textos escolares, el libro del estudiante no ayudaría al aprendizaje en los siguientes casos:

* Cuando el docente lo utiliza mecánicamente.

* Cuando no lo adapta a las capacidades de los alumnos.

* Cuando el texto es ininteligible para el estudiante.

De ahí la importancia de considerar, por igual, tanto los elementos didácticos propios del texto como la capacidad de discernimiento del docente para el uso de este material, lo cual se evidencia en el hecho de que el uso de un mismo texto puede generar diferentes resultados en diferentes aulas, de acuerdo con las distintas prácticas de los maestros (Zapata, 2006).

En este trabajo nos enfocamos exclusivamente en el texto como ayuda y guía en la enseñanza de la biología.

\section{Materiales y métodos}

El presente artículo es el resultado de un ejercicio realizado para el proyecto del primer trimestre del 2009 del Seminario Tendencias y Lineamientos Pedagógicos y Contemporáneos de la Biología, que hace parte del programa de Especialización en Enseñanza de la Biología de la Universidad Pedagógica Nacional.

Se seleccionó un libro de ciencias naturales de grado noveno de la editorial Norma, cuyo título es Viajeros 9 Ciencias. Este texto hace parte de una colección de amplio uso en el entorno educativo colombiano, según lo comentado en las secciones del Seminario mencionado anteriormente.

Para el análisis del mismo, se utilizó como referente el sistema de categorías de análisis, establecido en la tesis de Zulma Zapata (2006, pp. 87-88), el cual aparece esquematizado a continuación: 


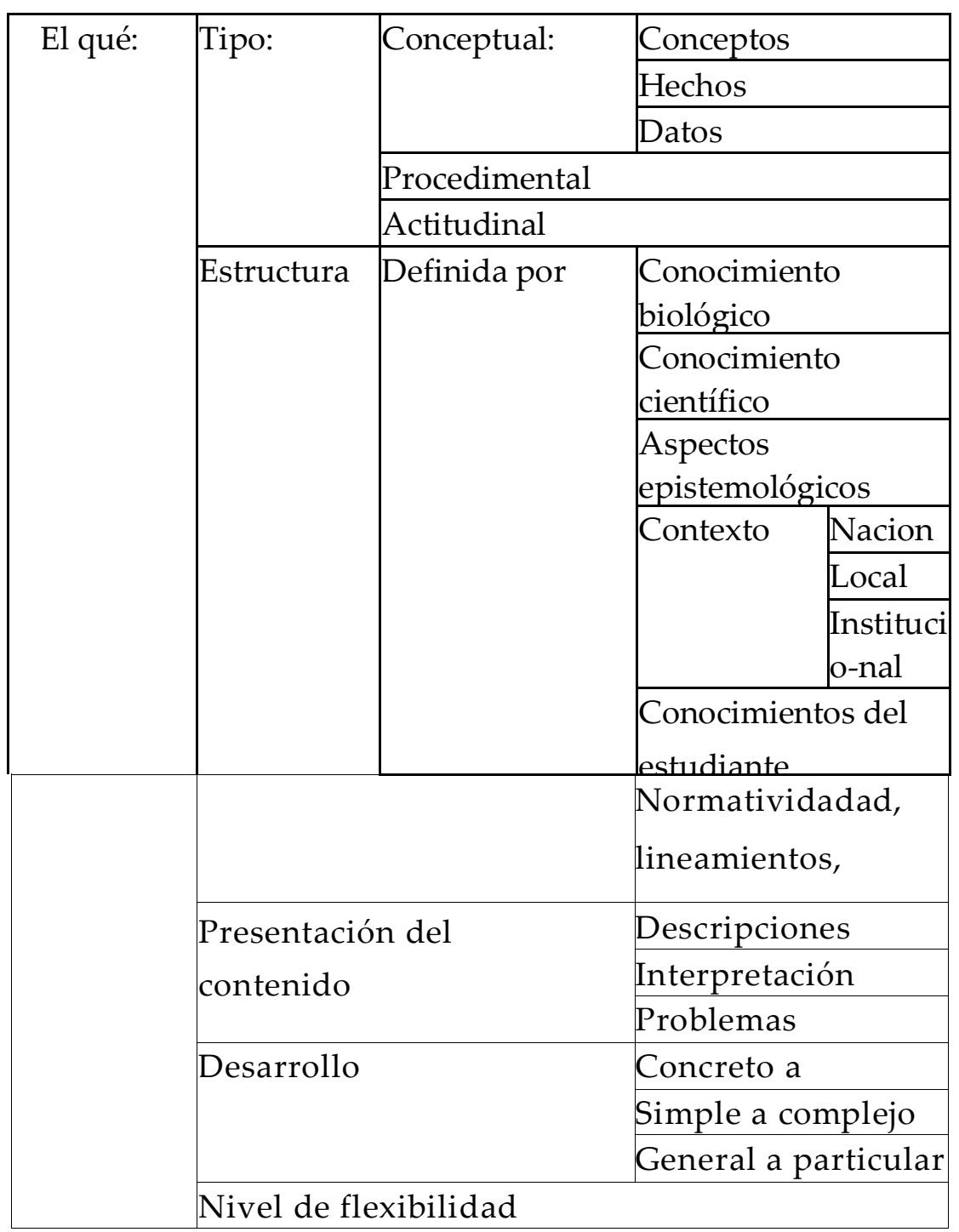

\begin{tabular}{|l|l|}
\hline Diagram & La presentación tiene secuencia de los eventos \\
as e & Aplicabilidad para la comprensión del texto \\
\hline ilustracio & Significatividad en la comprensión de los contenidos \\
nes & Estimulación de las construcciones mentales \\
\hline & Coherencia y correspondencia con el texto \\
\hline & Explicación y descripción de la situación presentada \\
\hline
\end{tabular}




\begin{tabular}{|c|c|c|c|c|}
\hline & \multicolumn{4}{|c|}{$\begin{array}{l}\text { Distribución homogénea (contenidos del texto } \\
\text { son similares a las imáoenes) } \\
\text { Información de la leyenda } \\
\end{array}$} \\
\hline \multicolumn{5}{|c|}{ Secuencia } \\
\hline \multicolumn{5}{|c|}{ Distribución de contenidos } \\
\hline \multicolumn{5}{|c|}{ Conceptos estructurantes } \\
\hline \multicolumn{5}{|c|}{ Integración } \\
\hline \multirow{4}{*}{\multicolumn{3}{|c|}{$\begin{array}{l}\text { Complejidad de la } \\
\text { representación }\end{array}$}} & \multicolumn{2}{|c|}{ Cuadros sinópticos } \\
\hline & & & \multicolumn{2}{|c|}{ Mapas conceptuales } \\
\hline & & & \multicolumn{2}{|c|}{ Redes conceptuales } \\
\hline & & & \multicolumn{2}{|c|}{ Otras } \\
\hline \multicolumn{5}{|c|}{ Niveles de profundidad } \\
\hline \multicolumn{5}{|c|}{ Actualidad } \\
\hline \multicolumn{5}{|c|}{ Rigurosidad de la información } \\
\hline \multicolumn{5}{|c|}{ Relaciones técnico- prácticas } \\
\hline \multirow{14}{*}{\multicolumn{2}{|c|}{ El cómo }} & \multirow{2}{*}{\multicolumn{3}{|c|}{$\begin{array}{l}\text { Niveles de significatividad (referente de } \\
\text { anrendizaie } \\
\text { Grado de apertura }\end{array}$}} \\
\hline & & & & \\
\hline & & \multicolumn{3}{|c|}{ Implicación de los estudiantes } \\
\hline & & \multicolumn{3}{|c|}{ Demanda cognitiva } \\
\hline & & \multirow{4}{*}{\multicolumn{2}{|c|}{$\begin{array}{l}\text { Evaluación del } \\
\text { aprendizaje }\end{array}$}} & Quién \\
\hline & & & & Cómo \\
\hline & & & & Para qué \\
\hline & & & & Cuándo \\
\hline & & \multirow{6}{*}{\multicolumn{2}{|c|}{$\begin{array}{l}\text { Características de } \\
\text { las } \\
\text { actividades (tipo) }\end{array}$}} & Dirigidas \\
\hline & & & & Informativas \\
\hline & & & & Experimentales \\
\hline & & & & Problemas \\
\hline & & & & Juegos \\
\hline & & & & Actividades \\
\hline & & \multirow{3}{*}{\multicolumn{2}{|c|}{ Estructura }} & Analogías \\
\hline & & & & Ejemplos \\
\hline & & & & Metáforas \\
\hline \multirow{5}{*}{\multicolumn{2}{|c|}{ Diseño }} & \multirow{2}{*}{\multicolumn{2}{|c|}{ Diagramación }} & Lenguaje icónico \\
\hline & & & & Tipo de letra \\
\hline & & \multicolumn{3}{|c|}{ Presentación de los contenidos (presenta la } \\
\hline & & \multicolumn{3}{|c|}{$\begin{array}{l}\text { Wnidad formado indina } \\
\text { Lecturas empleadas }\end{array}$} \\
\hline & & \multicolumn{3}{|c|}{ Número de páginas de la unidad } \\
\hline
\end{tabular}




\begin{tabular}{|l|l|}
\hline \multirow{4}{*}{} & Ubicación en el texto de la unidad \\
\cline { 2 - 2 } & Información bibliográfica \\
\cline { 2 - 2 } & Glosario \\
\cline { 2 - 2 } & Resumen \\
\hline \multirow{2}{*}{ Gestión de } & Materiales \\
\cline { 2 - 2 } recursos & Tiempo \\
\cline { 2 - 2 } & Espacio \\
\hline
\end{tabular}

\section{Resultados y discusión}

En cuanto a los contenidos presentados (el qué), encontramos que los tres tipos de contenidos aparecen en forma equilibrada en el texto. Dentro de los contenidos conceptuales algunos de los conceptos fundamentales para la comprensión del tema son resaltados, en negrilla, e incluidos en un glosario al final del libro. Como ejemplos tenemos los siguientes:

\begin{tabular}{|c|l|}
\hline Página & \multicolumn{1}{|c|}{ Conceptos fundamentales } \\
\hline 128 & diversidad, especie \\
\hline 129 & fósiles, creacionismo, evolución \\
\hline 130 & adaptación, demografía \\
\hline 131 & selección natural, especiación. \\
\hline 135 & taxonomía, sistemática \\
\hline
\end{tabular}

Entre los hechos presentados en el texto, se destacan algunos eventos históricos,con una serie de datos específicos que requerirían, según Pozo ( 1992), de un proceso de repetición para su aprendizaje literal o memorístico. Éstos incluyen las contribucioens de Buffon yLamarck al desarrollo del concepto de evolución (p. 129), el viaje de Darwin a las islas Galápagos a la edad de 22 años a bordo del Beagle, la obra de Malthus sobre el crecimiento poblacional (p. 130), la identificación independiente del proceso de selección natural por Wallace, las ideas de Mendel y su integración en la síntesis evolutiva moderna (p. 131), la tectónica de placas propuesta por Wegener (pp.132-133), el surgimiento de la taxonomía con Aristóteles, los trabajos de clasificación y nomenclatura de Linneo (p. 135), y los diferentes sistemas de clasificación de los reinos, en los que se mencionan los trabajos de Haeckel, Copeland, Wittaker y Woose (p. 136).

Como datos, se pueden citar los mecanismos de aislamiento descritos brevemente en la página 128, la publicación del Diario del viaje del Beagle y las diferentes formas del pico asociadas al tipo de alimentación y hábitat (p. 130), la evidencia fósil (Mesosaurus, 
Glossopteris, etc.) que apoya la teoría de Wegener y tipos de comunidades vegetales de Colombia (pp. 132-133), los nombres científicos de algunas especies de primates y homínidos, y sus relaciones filogenéticas (p. 134), una enumeración de los aportes de Linneo (p. 135), los nombres de los grupos de organismos y algunas de las características usadas para su clasificación (p. 136), una corta explicación del método de datación mediante carbono 14 (p. 141) y una descripción de las profesiones de Astrobiología y Taxonomía (p. 144).

En relación con los contenidos procedimentales el tema de diversidad inicia con un Taller de exploración (p. 127) en el cual se pide a los estudiantes que recolecten una muestra de agua estancada y una muestra de suelo, para observar y dibujar diferentes tipos de organismos, con la ayuda de un estereoscopio o una lupa; luego, deben comparar los dos ecosistemas y analizar los resultados en torno a dos preguntas orientadoras. En la página 128, se formula un problema abierto en la leyenda de la figura 6.1. Luego, en la sección titulada La clasificación de los seres vivos (p. 135), aparece un trabajo individual en el que dada estudiante debe obtener información sobre la evolución humana para elaborar una línea de tiempo que describa este proceso. En la sección denominada Investigación de aplicación (p. 138-139) se plantea un trabajo práctico de campo y de laboratorio en taxonomía vegetal, en el que los estudiantes recolectan muestras de plantas, describen sus características a través de una matriz de datos y las identifican con la ayuda de las claves que aparecen citadas en la bibliografía recomendada. En este caso, también se dirige el análisis de los resultados por medio de una serie de preguntas orientadoras. Por último, en la página 139 el texto presenta a los estudiantes el problema de crear una clave para identificar plantas medicinales (el cual incluye un esquema de investigación científica), reforzando así las destrezas y conocimientos que los alumnos adquieran con la ejecución de la actividad previa. Además de todo lo anterior, el texto también incluye un apartado titulado: Evalúa tus competencias (p. 140) con una serie de problemas abiertos en los cuales los estudiantes deben aplicar todo lo aprendido.

Dentro de los contenidos actitudinales se destacan los siguientes:

\begin{tabular}{|c|l|}
\hline Página & \multicolumn{1}{|c|}{ Ejemplo de contenido actitudinal } \\
\hline 126 & $\begin{array}{l}\text { Antes de dar inicio al tema el texto } \\
\text { muestra un espacio denominado Mis } \\
\text { preguntas sobre el tema, en el cual se } \\
\text { genera cierta expectativa en el estudiante } \\
\text { y, de paso, le da la oportunidad de sacar a } \\
\text { la luz su propio saber sobre el tópico de } \\
\text { diversidad. }\end{array}$ \\
\hline 128 & \begin{tabular}{l} 
La pregunta que aparece en la figura 6.1, \\
\hline
\end{tabular} \\
\hline
\end{tabular}




\begin{tabular}{|c|c|}
\hline & $\begin{array}{l}\text { más que un medio de evaluación es una } \\
\text { forma de implicar e interesar al educando } \\
\text { en el tema. }\end{array}$ \\
\hline 132 & $\begin{array}{l}\text { Al inicio de la explicación sobre las placas } \\
\text { tectónicas se acude a una situación } \\
\text { cotidiana para el alumno para llamar su } \\
\text { atención y facilitar su comprensión: "Si } \\
\text { observas un mapa actual de la Tierra, puedes } \\
\text { notar la coincidencia que existe entre América } \\
\text { del Sur y África. Son dos continentes que } \\
\text { coinciden casi como piezas de un } \\
\text { rompecabezas". }\end{array}$ \\
\hline 135 & $\begin{array}{l}\text { En este trabajo individual se da cierta } \\
\text { libertad a los estudiantes para que } \\
\text { elaboren la línea de tiempo, lo cual hace } \\
\text { más interesante esta tarea: "Completa la } \\
\text { línea con información sobre distintas etapas } \\
\text { importantes del desarrollo de la humanidad, } \\
\text { como los viajes espaciales, el invento del } \\
\text { computador, el Internet y otros que te parezcan } \\
\text { importantes". }\end{array}$ \\
\hline 139 & $\begin{array}{l}\text { En la sección Ahora, hazlo tú, no sólo se } \\
\text { refuerza lo aprendido en el problema } \\
\text { previo de clasificación, sino que se intenta } \\
\text { mostrar la utilidad de ese tipo de } \\
\text { conocimiento, con el fin de generar interés } \\
\text { en las hierbas y sus usos medicinales, por } \\
\text { parte del estudiante. De esta forma, se le } \\
\text { otorga un papel protagónico como sujeto } \\
\text { social: "Imagina que te han contratado para } \\
\text { diseñar un sistema de clasificación que ayude a } \\
\text { los vendedores de plantas medicinales de } \\
\text { alguna región del país a clasificar dichas } \\
\text { plantas". }\end{array}$ \\
\hline 143 & $\begin{array}{l}\text { En este espacio denominado Mi rincón, al } \\
\text { educando se le incita a reflexionar sobre su } \\
\text { experiencia de aprendizaje sobre la } \\
\text { diversidad, lo cual le permitiría } \\
\text { intercambiar sus puntos de vista con otros } \\
\text { compañeros y generar propuestas a la } \\
\text { diferentes problemáticas entorno a este } \\
\text { tema. }\end{array}$ \\
\hline 144 & En la sección de Profesiones de la ciencia, el \\
\hline
\end{tabular}




\begin{tabular}{|l|l|}
\hline texto muestra dos posibilidades de trabajo \\
a los alumnos, las cuales podrían \\
despertar en algunos de ellos vocaciones \\
hacia el estudio más profundo de esas \\
disciplinas. Sin embargo, consideramos \\
que esta sección se queda corta en ese \\
propósito, ya que carece del elemento \\
biográfico que le da un carácter más \\
humano a la ciencia y que aporta \\
experiencias de vida valiosas para los \\
lectores.
\end{tabular}

Teniendo en cuenta la definición de Sarabia (1992: 137) de las actitudes como “... tendencias o disposiciones adquiridas y relativamente duraderas a evaluar de un modo determinado un objeto, persona, suceso o situación y a actuar en consonancia con dicha evaluación", vemos en todos estos ejemplos en interés de los autores por generar en los estudiantes una actitud de aprecio e interés por el tema presentado.

La estructura de los contenidos del tema, está definida principalmente por los aspectos biológicos más importantes que giran en torno a la diversidad. Así dominan las actividades de historia natural (pp. 127, 138 y 139), lo cual no es de extrañar, ya que la observación y la comparación juegan un papel clave a la hora de describir la diversidad y son las más adecuadas para aproximar a los alumnos a este fenómeno biológico; también, se muestran algunos nombres científicos de algunos géneros ( $p$. 132) y especies (pp. 134-135), familiarizando al lector con la nomenclatura biológica. Como ya se mencionó más arriba, se presentan algunos de los conceptos fundamentales que les permiten a los estudiantes comprender mejor el proceso de generación de las especies y su clasificación, y en dicha presentación se incluye tanto algunos aspectos históricos como algunos de los últimos desarrollos, tal como se aprecia en la sección titulada: Las categorías de clasificación (pp. 135- 136). Además, estos contenidos biológicos están organizados de tal forma que el lector va relacionando los conceptos estructurantes relevantes, lo cual le permite comprender de forma integral la diversidad biológica. Lo anterior, se complementa con una explicación clara que es reforzada con varias ilustraciones que refuerzan el discurso, siendo estos dos aspectos un reflejo de la intención de los autores de adecuar este discurso al nivel educativo de los estudiantes.

La estructuración de los contenidos del tema de diversidad, también considera otros conocimientos científicos tales como la geología (p. 132), la geografía (p. 133) y la paleontología (p. 141) que permiten comprender los orígenes y el desarrollo histórico de la diversidad. Otra ventaja de este texto es el hecho de que muestra, al alumno, en ciertas 
secciones (pp. 129-131 y 135-136), que la ciencia es una empresa humana en continuo desarrollo que no tiene verdades absolutas, ni un único método correcto, tal como se aprecia en el siguiente extracto que refleja la problematización de la actividad científica: "Vemos entonces que en la biología hay opiniones diferentes apoyadas por hechos y que la comunidad científica no siempre se pone de acuerdo, pues la ciencia cambia a medida que los avances tecnológicos lo permiten" (p. 136). En esta frase faltaría agregar que la ciencia también cambia con el desarrollo de nuevos conceptos.

En cuanto a los aspectos epistemológicos e históricos que definen la estructura de los contenidos, y cuya importancia y relación con la enseñanza han sido analizadas por varios autores, tales como Gagliardi y Giordan (1986), Gagliardi (1988) Gil-Pérez (1993), entre otros, cabe destacar las secciones históricas comentadas anteriormente en las que se describe el desarrollo de la teoría de la evolución y su incorporación en la síntesis evolutiva moderna (pp. 129-131), así como las diferentes propuestas de clasificación de los organismos (pp. 135- 136), con lo cual se muestra al estudiante la forma en que el desarrollo de los conceptos es afectado por el ámbito científico del momento, el cual incluye, según Gil-Pérez (1993), las dificultades que enfrentan los científicos y las condiciones que facilitan la resolución de problemas; de ahí su gran valor didáctico. Sin embargo, en estos relatos históricos encontramos los siguientes errores: en el primero se atribuye la teoría de la síntesis evolutiva moderna únicamente a Theodosius Dobzhanzky (p. 131), dejando de lado a figuras importantes en su desarrollo, tales como a Ernst Mayr, George Gaylord Simpson, R. A. Fisher, J.B.S. Haldane, Sewall Wright, Thomas Hunt Morgan, William Donald Hamilton, Cyril Darlington, Julian Huxley, y G. Ledyard Stebbins (Gould, 2002), en una declarada omisión histórica. El segundo error es de tipo conceptual y consiste en la confusión en el texto entre la nomenclatura y el sistema de clasificación propuestos por Linneo, los cuales son presentados como una sola construcción conceptual en la siguiente oración: "Linné estableció un sistema jer\{rquico y binario" (p. 135). El tercer error, también de tipo conceptual, corresponde a la inclusión de las bacterias entre los eucariotas, cuando se explica el sistema de clasificación de Woese, en una clara contradicción con lo que se venía exponiendo; esto se observa en las siguientes líneas: “Posteriormente, en 1990, Woese planteó la clasificación en tres dominios: Bacteria, Archea y Eucharia. Woese argumenta entonces que, aunque una planta, un animal un hongo y una bacteria son muy diferentes en apariencia, a nivel celular comparten el hecho de ser eucariotas, tener la misma conformación celular y un núcleo definido. Estas razones apoyan el hecho de incluir esto cuatro reinos bajo el dominio Eucaria" (p. 136).

En el tratamiento del tema de diversidad se tiene en cuenta, en cierta medida, el contexto local de los estudiantes en los trabajos prácticos (pp. 127, 138-139), ya que se proponen actividades en entornos cotidianos para los estudiantes tales como, humedales, parques, jardines y plazas de mercado más cercanas. También, considera el contexto nacional 
cuando presenta en la página 133 los diferentes ecosistemas presente en nuestro territorio.

La estructura del libro le permite al docente dar cuenta de los preconceptos de los estudiantes en los espacios titulados: Mi preguntas sobre el tema (vg. p. 126), así como las actitudes frente a lo enseñado en la sección Mi rincón (p. 143).

Los autores de este texto tienen en cuenta la normatividad consignada en los estándares curriculares propuestos por MEN para el área de ciencias naturales y educación ambiental. Así, de acuerdo con la bitácora para el docente (Arbeláez et al., 2009: A12), el tema de diversidad tiene una estructura que lleva al alumno a alcanzar los siguientes logros:

- “Identifico los argumentos que sustentan las principales teorías sobre la evolución de los seres vivos.

- Identifico los criterios empleados para clasificar a los seres vivos.

- Establezco relaciones entre el ambiente y la diversidad de los organismos".

Así, al observar el contenido de este tema, se ve una coherencia inequívoca entre el discurso pedagógico y los objetivos o logros propuestos.

Con respecto a la presentación de los contenidos predominan las descripciones de los diferentes conceptos que articulan el tema de diversidad, muchos de los cuales son nuevos para los estudiantes. Dentro de estos conceptos se incluyen aquellos que no pertenecen al núcleo conceptual, pero que permiten una mejor comprensión del mismo, tal como se aprecia en la explicación de los mecanismos de aislamiento reproductivo (p. 128) que cumplen la función de reforzar el concepto de especie biológica, previamente definido. También hay pasajes explicativos como el de la sección sobre las placas tectónicas ( $p$. 132), en el que se presenta la teoría formulada por Wegener para interpretar la distribución de los fósiles.

Como ejemplo de problemas o interrogantes no retóricos en el tema de diversidad se encuentra la sección Los virus ¿seres vivos? (p. 136) en donde se plantea el problema de la posición de los virus con respecto a los organismos, aunque no cumple a cabalidad con la finalidad de poner a prueba las ideas de los estudiantes o generar en ellos interés, pues lo resuelve de forma simplista en lugar de dejar abierta la controversia.

En cuanto al desarrollo de los contenidos podemos ver que va de lo concreto a lo 
abstracto y de lo familiar a lo desconocido, pues, previamente a su presentación, se encuentra la sección Mis preguntas sobre el tema (p. 126) que permite a los alumnos hacer un cuestionamiento previo de sus propios conocimientos, despertando su interés desde sus experiencias personales. Luego se introducen algunos contenidos en el Taller de exploración (p. 127) a través de un trabajo práctico con elementos tomados de la vida cotidiana, y después se presentan los elementos teóricos del caso, en los cuales se describen los conceptos más generales, los cuales se explican a través de ejemplos y de datos particulares.

Con relación al nivel de flexibilidad en la estructura de los contenidos, consideramos que podría modificarse el orden en el que se presentan los contenidos (por ejemplo, iniciando con la sección de clasificación), aunque la secuencia que aparece en el texto es adecuada para facilitar la comprensión del estudiante.

Los diagramas e ilustraciones cumplen con la función de ilustrar y complementar la exposición escrita, lo cual se refleja en una correspondencia y coherencia con el texto elevada (distribución homogénea). Además, abarcan aproximadamente el $50 \%$ del espacio destinado al tema, siendo este un indicativo del interés de los autores por dar mayor cabida a los contenidos actitudinales en la presentación del tema, a través de una diagramación atractiva. Algunas (figuras 6.1 y 6.6) estimulan la representación de construcciones mentales a través de preguntas en las leyendas o de secuencias (figuras 6.2, 6.4 y 6.5). Entre los errores detectados en las figuras destacamos los siguientes:

\begin{tabular}{|c|l|}
\hline Figura del texto & \multicolumn{1}{|c|}{ Descripción del error } \\
\hline 6.5 & $\begin{array}{l}\text { Los nombres científicos no están en letra } \\
\text { cursiva, lo cual va en contra de las } \\
\text { recomendaciones de la nomenclatura } \\
\text { biológica.Además, la leyenda no explica } \\
\text { la ilustración. }\end{array}$ \\
\hline 6.7 & $\begin{array}{l}\text { En la microfotografía no aparece una } \\
\text { escala de medidas o la magnificación. }\end{array}$ \\
\hline
\end{tabular}

Por último, recomendamos colocar en la figuras 6.1, 6.3, 6.4 y 6.6 y en la ilustración de la página 41 , los nombres comunes y/o científicos de los ejemplares representados.

Tal como se mencionó previamente, consideramos que la secuencia de los contenidos que se presentan es adecuada para facilitar la construcción de una visión más compleja y holística de la diversidad, en la que se aprecia la integración de varias disciplinas y algunas implicaciones de este conocimiento. Dicha secuencia se esquematiza a continuación: 


\begin{tabular}{|c|c|c|}
\hline TÍTULO & $\begin{array}{l}\text { SUBTÍTULO } \\
\text { (segundo nivel) }\end{array}$ & $\begin{array}{l}\text { Subtitulo } \\
\text { (segundo nivel) }\end{array}$ \\
\hline \multicolumn{3}{|l|}{ MES PREGUNTAS SOBRE EL TEMA } \\
\hline \multicolumn{3}{|c|}{ TALLER DE EXPLORACIÓN: UN MUNDO DIVERSO } \\
\hline \multirow{4}{*}{$\begin{array}{l}\text { HEMOS CAMBIADO } \\
\text { DESDE NUESTRA } \\
\text { APARICIÓN SOBRE LA } \\
\text { TIERRA }\end{array}$} & ELCONCEPTO DE & Mecanismos de aislamiento \\
\hline & ESPECIE & Reproductivo y especiación. \\
\hline & \multirow[t]{2}{*}{$\begin{array}{l}\text { TEORÍA DE LA } \\
\text { EVOLUCIÓN }\end{array}$} & $\begin{array}{l}\text { Las primeras explicaciones } \\
\text { evolutivas }\end{array}$ \\
\hline & & $\begin{array}{l}\text { Hacia una teoría moderna de } \\
\text { evolución }\end{array}$ \\
\hline \multicolumn{3}{|c|}{$\begin{array}{l}\text { LAS PLACAS TECTÓNICAS Y LA DIVERSIDAD DE LAS ESPECIES EN LA } \\
\text { TIERRA }\end{array}$} \\
\hline \multicolumn{3}{|c|}{ LA EVOLUCIÓN DE LA ESPECIE HUMANA } \\
\hline \multirow{3}{*}{$\begin{array}{l}\text { LA CLASIFICACIÓN DE LOS SERES } \\
\text { VIVOS }\end{array}$} & \multicolumn{2}{|c|}{ EL SISTEMA UNIVERSAL DE CLASIFICACIÓN } \\
\hline & \multicolumn{2}{|c|}{ LAS CATEGORÍAS DE CLASIFICACIÓN } \\
\hline & \multicolumn{2}{|c|}{ LOS VIRUS ¿SERES VIVOS? } \\
\hline \multicolumn{3}{|c|}{ PARA RECORDAR (repaso de ideas y conceptos) } \\
\hline \multirow[t]{2}{*}{ INVESTIGACIÓN DE APLICACIÓN } & \multicolumn{2}{|c|}{$\begin{array}{l}\text { RESUELVE ESTE PROBLEMA ¿CÓMO } \\
\text { REFERENCIAR SERES VIVOS? }\end{array}$} \\
\hline & \multicolumn{2}{|c|}{ AHORA, HAZLO TÚ } \\
\hline \multicolumn{3}{|c|}{$\begin{array}{l}\text { EVALÚA TUS COMPETENCIAS (problemas de aplicación de los } \\
\text { conceptos expuestos) }\end{array}$} \\
\hline \multicolumn{3}{|c|}{ CIENCIA, TECNOLOGÍA Y SOCIEDAD: DE REGRESO AL PASADO } \\
\hline PRUEBA SABER (sección de evaluaci & & \\
\hline
\end{tabular}

La distribución de los contenidos es más bien equilibrada, con los componentes procedimentales, actitudinales y conceptuales adecuadamente representados, aunque en algunas secciones predominan unos más que otros. 
Además, es importante anotar que en esa sección se incluyen algunos conceptos estructurantes que, según Gagliardi (1986), ocupan un lugar central dentro de la biología y por lo tanto son fundamentales en la enseñanza de esta ciencia. Entre los conceptos estructurantes que se encuentran en el texto se destacan los de diversidad, evolución, selección natural y especie, los cuales constituyen el eje articulador del discurso. También, hay otros conceptos importantes que permiten comprender los primeros, tales como aislamiento reproductivo, fósil, adaptación, clasificación, sistemática, taxonomía, por solo nombrar los más importantes.

La integración de los contenidos es exitosa en el desarrollo del tema, pues, tal como se comentó más arriba, los contenidos tienen una secuencia coherente y una distribución equilibrada que da unidad al discurso.

Al analizar la complejidad en la presentación del tema de diversidad, encontramos los siguientes elementos didácticos:

\begin{tabular}{|c|l|}
\hline Página & \multicolumn{1}{c|}{$\begin{array}{c}\text { Ejemplos de elementos didácticos de } \\
\text { mayor complejidad }\end{array}$} \\
\hline 127 & $\begin{array}{l}\text { Se propone una tabla comparativa y un } \\
\text { diagrama de Venn como medio } \\
\text { para sistematizar la información obtenida } \\
\text { por los estudiantes en el taller } \\
\text { de exploración. }\end{array}$ \\
\hline 130 & $\begin{array}{l}\text { La figura 6.3 requiere de un análisis } \\
\text { detenido por parte de los alumnos } \\
\text { para comprender el fenómeno de la } \\
\text { adaptación; probablemente requiera } \\
\text { de una explicación adicional del profesor. }\end{array}$ \\
\hline 131 & $\begin{array}{l}\text { La figura 6.4, es un caso equivalente al } \\
\text { anterior y tiene el potencial de ser usada } \\
\text { como una actividad de análisis en clase. }\end{array}$ \\
\hline 134 & $\begin{array}{l}\text { La figura 6.5 representa la filogenia de los } \\
\text { primates y homínidos, que carece de una } \\
\text { leyenda explicativa y de una aclaración en } \\
\text { el texto principal, por lo cual el docente } \\
\text { tiene que dar una explicación en clase de } \\
\text { su contenido. }\end{array}$ \\
\hline En la sección de conceptos aparece un \\
\hline
\end{tabular}




\begin{tabular}{|c|l|}
\hline & $\begin{array}{l}\text { ejercicio de repaso a través de un mapa } \\
\text { conceptual, con lo cual se pretende ir más } \\
\text { allá de la simple memorización, } \\
\text { reforzando las relaciones existentes entre } \\
\text { los mismos. }\end{array}$ \\
\hline 138 & $\begin{array}{l}\text { Se presenta una tabla para sistematizar los } \\
\text { datos obtenidos de las plantas } \\
\text { recolectadas. }\end{array}$ \\
\hline 140 & $\begin{array}{l}\text { En la sección Evalúa tus competencias, se } \\
\text { le pide al lector interpretar la ilustración y } \\
\text { la tabla para resolver algunas de las } \\
\text { preguntas formuladas. }\end{array}$ \\
\hline $141-142$ & $\begin{array}{l}\text { En esta evaluación el estudiante tiene que } \\
\text { interpretar gráficos y tablas } \\
\text { para completar esta actividad. }\end{array}$ \\
\hline
\end{tabular}

Los niveles de profundidad de los contenidos procedimentales, actitudinales y conceptuales son aproximadamente equivalentes, tal como se explicó anteriormente. En el caso del último tipo de contenidos, encontramos que el tema de diversidad se presenta en forma general como una panorámica global que gira en torno a unos cuantos conceptos estructurantes, reforzados por algunos datos o contenidos puntuales; así, por ejemplo en la sección de clasificación de los seres vivos no se detallan los diferentes grupos de organismos. Esto es compensado, en parte, con los trabajos prácticos y con los problemas y consultas individuales. Además, en los contenidos procedimentales y actitudinales se busca que el estudiante avance un poco más en el desarrollo del tema, a través del planteamiento de problemas abiertos de mayor complejidad y que tengan relación con sus experiencias cotidianas (vg. p. 138-139).

En el texto se presentan contenidos disciplinares más bien actualizados, especialmente en la sección de clasificación (p. 136) en la que se describe la influencia de los avances científicos, tales como las técnicas en biología molecular, en el desarrollo de los sistemas de clasificación, y muestra las controversias científicas derivadas de estos adelantos, lo cual rompe con el esquema común de la ciencia como una actividad estática y absoluta.

Como ejemplos de rigurosidad se tienen los trabajos prácticos sugeridos en el libro, en los cuales se proponen metodologías de trabajo organizadas, inspiradas en el método científico y en su utilidad didáctica. En este caso, vale la pena destacar la sección de Investigación de aplicación (pp. 138-139), en la que se aproxima a los alumnos al trabajo en taxonomía con plantas, a través de la ejecución de algunas de las actividades propias 
de los botánicos, tales como la elaboración de matrices de datos con las principales características de las plantas, la identificación de los ejemplares recolectados con la ayuda de claves, la consulta de algunos textos especializados, entre otras.

Anteriormente, se describió cómo los aspectos epistemológicos e históricos definen la estructura de los contenidos, sin embargo, dichos aspectos se constituyen en contenidos por sí mismos, con un gran valor didáctico. Lo anterior, teniendo en cuenta el comentario de Hodson (1998) sobre el fenómeno señalado por varios profesores de ciencia respecto a la descripción tradicional (positivista) de la actividad científica, la cual se caracteriza por una visión engañosa de lo que es la ciencia y de cómo los científicos trabajan. Desde este punto de vista, el conocimiento científico es descubierto por especialistas objetivos, el cual conforma un cuerpo de conocimientos que el estudiante debe aprender. En el discurso se rompe con ese esquema mostrando brevemente el desarrollo histórico y epistemológico de la teoría de la evolución y de los sistemas de clasificación, sacando a la luz algunas de las principales rupturas, obstáculos y paradigmas propios de la génesis de estos conceptos. De esta forma, se destaca la naturaleza provisional y contingente de la ciencia en el currículo de ciencias naturales y educación ambiental.

Las relaciones teórico-prácticas que observamos en el tratamiento del tema son de complementariedad, ya que en los trabajos prácticos se aplican y refuerzan los contenidos conceptuales. En general, se aprecia cómo en las actividades propuestas, las habilidades, destrezas y técnicas que el alumno requiere para su desarrollo son explicadas en el texto.

Con relación a las características de las actividades (el cómo) el tema tiene un alto nivel de significatividad, ya que adapta el formato y la presentación de los contenidos a las características de los estudiantes, lo cual se aprecia en aspectos tan diversos como el lenguaje empleado, las numerosas figuras, los trabajos prácticos, la indagación de las ideas previas, la incitación a la reflexión, entre otros.

El grado de apertura de las actividades es en general bastante alto, con muchas prácticas, preguntas y problemas abiertos, en los cuales se indican algunos lineamientos generales, pero el alumno tiene la libertad de decidir algunos de los aspectos de la actividad. Sólo observamos preguntas cerradas de selección múltiple en la sección Prueba saber (pp. 142-143).

Tal como se comentó con anterioridad, el texto ofrece a los educandos diferentes oportunidades para autorregular su proceso de aprendizaje (vg., la sección Mi Rincón de 
la página 143), por lo cual consideramos que el discurso tiene un buen nivel de implicación de los estudiantes.

El texto presenta una gran demanda cognitiva en las secciones correspondientes a los trabajos prácticos (pp. 127 y 138-139), pues estos requieren que el alumno sistematice la información y consulte diferentes fuentes. Además, estas actividades estimulan el desarrollo de diferentes habilidades procedimentales y la integración de varios conceptos. Así mismo, en la sección denominada Evalúa tus competencias (p. 140) se formulan problemas abiertos que requieren la interpretación de figuras y la aplicación de los contenidos estudiados.

En relación con la evaluación del aprendizaje, se observa que en la presentación del tema hay preguntas abiertas en las que se evalúan la comprensión y aplicación de algunos conceptos biológicos fundamentales (p. 140) o el repaso de los mismo (p. 137). También se encuentra hacia el final del mismo una serie de problemas de análisis, pero planteados a través de preguntas de selección múltiple, con respuestas únicas y cerradas, y en la sección Mi rincón (p. 143) un espacio de autoevaluación y reflexión metacognitiva. Vale la pena anotar que en el texto no se pregunta al alumno acerca de hechos o datos puntuales. Todo lo anterior, permite afirmar que la intención de la evaluación es propiciar un aprendizaje significativo de los conceptos estructurantes de la biología que están relacionados con la diversidad.

Las características de las actividades observadas en el tema de diversidad incluyen actividades dirigidas y experimentales en las que predomina la observación, la descripción de organismos (a través de una recolección sistemática de caracteres morfológicos) y existe una relación entre la práctica y la teoría (pp. 127, 138-139); y la resolución de problemas abiertos (pp. 139, 140) y cerrados (pp. 142-143). Dentro del contenido del tema de diversidad no hay juegos, pero en el libro si encontramos dos juegos al inicio de los entornos biológico y físico. Por último se plantean actividades complementarias de consulta en las figuras 6.1 y 6.6 y lecturas adicionales sobre conceptos de geología, geografía y de paleontología (pp. 132-133, 141), que refuerzan la comprensión del fenómeno biológico de diversidad.

Con respecto a la estructura del texto, tenemos como ejemplo de analogía la siguiente frase de la página 132: "Si observas un mapa actual de la Tierra, puedes notar la coincidencia que existe entre América del Sur y África. Son dos continentes que coinciden como piezas de un rompecabezas". Además hay varios ejemplos textuales (pp. 128, 129, 130, 135, 136) e icónicos (figuras 6.1, 6.2, 6.3, 6.4, 6.7; ilustraciones de las páginas $132-133,138,141,144)$ que complementan la explicación de los conceptos, facilitando su 
comprensión.

En este libro también es importante destacar el esmero en el diseño, lo cual, hace de este material didáctico un objeto más llamativo y accesible para el estudiante. De esta forma, observamos el uso frecuente del lenguaje icónico en el empleo de numerosas figuras y en la diagramación e ilustración del texto, con secciones claramente delimitadas en diferentes colores y tipos de letra, lo que facilita su reconocimiento y ubicación por parte del lector.

En cuanto a la presentación de los contenidos dentro del tema de diversidad, este no incluye índice temático alguno, sino que presenta directamente las actividades y contenidos, por lo cual el lector debe recurrir a la tabla de contenidos general que se encuentra en las primeras páginas del texto.

Las lecturas complementarias de esta sección del libro que están claramente separadas del resto de los contenidos, son las de Las placas tectónicas y la diversidad de las especies en la Tierra (pp. 132-133), De regreso al pasado (p. 141) y Profesiones de la ciencia (p. 144). Estas lecturas amplían la perspectiva del estudiante sobre la diversidad, mostrándole la naturaleza interdisciplinaria de las investigaciones en esta área de la biología y aportando ejemplos de algunas de las profesiones que se dedican a su estudio.

El número de páginas dedicadas al desarrollo de este tema es de 18. Dentro de ese espacio limitado, el concepto de especie ocupa una página, la teoría de la evolución se presenta en tres páginas y la sección de clasificación abarca dos páginas, mientras que otros conceptos complementarios que se explican en las lecturas ocupan cuatro páginas. Los contenidos procedimentales que se entremezclan con los conceptuales, se presentan en seis páginas y los contenidos actitudinales ocupan dos páginas.

El tema de diversidad hace parte de una unidad mayor, en la cual se explica el surgimiento de las especies en la tierra y se ubica después de un tema que trata sobre la formación del universo y la aparición de la vida en la tierra. Así, se aprecia que el tema analizado en este artículo se integra a la unidad siguiendo una estructura basada en el proceso histórico y evolutivo de la vida en nuestro planeta. Con respecto a la ubicación de esta unidad en el libro, ésta se encuentra estratégicamente localizada después de la exposición del método científico y del trabajo en ciencias, y de una unidad que presenta 
temas de genética y fisiología, con lo cual el lector llega más preparado para entender los conceptos de evolución y diversidad.

Otros aspectos del diseño del texto son los siguientes:

- La única información bibliográfica presente en el tema de diversidad es la que aparece en la sección de Investigación de aplicación (pp. 138-139), la cual es requerida para la realización de los trabajos prácticos propuestos en ese apartado.

- Tal como se explicó anteriormente, en el glosario que se encuentra hacia el final del libro se definen en forma escueta algunos de los conceptos desarrollados.

- En este material didáctico no encontramos resúmenes.

Finalmente, con respecto a la gestión de recursos del texto, se destaca la flexibilidad en el desarrollo de las actividades. Esto se puede ver en los trabajos prácticos propuestos, los cuales se caracterizan por usar materiales de fácil consecución y de costos bajos. Además, estas actividades se pueden distribuir en un número variable de sesiones de acuerdo con el tiempo disponible en las distintas instituciones educativas y no requieren de un espacio especial para su implementación, lo cual estimula a los docentes incluir estas prácticas en sus programas de enseñanza.

\section{Conclusiones}

Con base en el análisis anterior, podemos concluir lo siguiente:

* Los contenidos del tema de diversidad, se presentan en forma estructurada y coherente; además incluyen en forma equilibrada los contenidos de tipo procedimental, conceptual y actitudinal.

* Se detectaron algunos errores que pueden generar confusión en los estudiantes por lo cual deben ser corregidos urgentemente.

* Se aprecia el interés de los autores, en facilitar el aprendizaje significativo de los alumnos a través de una presentación llamativa y atractiva, con un lenguaje claro y varios contenidos actitudinales.

* Los contenidos se desarrollaron en un espacio bastante limitado, sin embargo, se logra una presentación equilibrada de los mismos.

* Las actividades prácticas tienen las ventajas de ser fáciles de implementar en las instituciones educativas colombianas, y de incorporar las experiencias cotidianas de los alumnos. 


\section{Agradecimientos}

Aprovechamos esta oportunidad para agradecer toda la colaboración que recibimos del profesor Edgar Valbuena de la Universidad Pedagógica Nacional, quien facilitó los contactos con la editorial, nos asesoró y nos proporcionó material bibliográfico de apoyo. También queremos agradecer especialmente a la Editora del Área de Ciencias Naturales y a la Editora del Área Científica, de ese grupo editorial, por suministrarnos el libro que fue objeto de análisis para la elaboración de este artículo.

\section{Bibliografía}

- Arbeláez E., F.; Carrillo C., E.; Castro G., N.; Gaviria de Gómez, M. \& Orjuela, M.A.(2009). Bitácora para el docente: Viajeros 9 ciencias. Bogotá: Norma.

- Arbeláez E., F.; Carrillo C., E.; Castro G., N.; Gaviria de Gómez, M. \& Orjuela, M.A.(2009). Viajeros 9 ciencias. Bogotá: Norma.

- Crisci, J.V. (1994). Biodiversity in the classroom. En: A call for action: Environmental education now and for a sustainable future. (Ed. K. Nakayama), pp. 161- 168.

- Crisci, J.V. (2001). La biodiversidad como recurso vital de la humanidad. Anales de la Academia Nacional de Agronomía y Veterinaria. Tomo LV: 256-269.

- Gagliardi, R. (1986). Los conceptos estructurantes en el aprendizaje por investigación.Enseñanza de las Ciencias, 4(1), 30 - 35.

- Gagliardi, R. (1988). Cómo utilizar la Historia de las Ciencias en la enseñanza de las Ciencias. Enseñanza de las Ciencias, 6(3), 291-296.

- Gagliardi, R.; Giordan, A. (1986). La historia de las ciencias: una herramienta para la enseñanza. Enseñanza de las Ciencias, 4(3), 253-258.

- Gil-Pérez, D. (1993) Contribución de la Historia y Filosofia de las Ciencias al desarrollo de un modelo de enseñanza/aprendizaje como investigación. Enseñanza de las Ciencias, 11(2), 197-212.

- Gould S.J. (2002). The Structure of Evolutionary Theory. Cambridge MA: Harvard University Press. 
- Hodson, D. (1998) Science fiction: the continuing misrepresentation of science in the school curriculum. Curriculum Studies, 6, 191-216.

- Pozo, J.I. (1994). El aprendizaje y la enseñanza de Hechos y Conceptos. En Coll et al. (Eds.), Los contenidos en la reforma: Enseñanza y aprendizaje de conceptos, procedimientos y actitudes (pp. 19-79). Madrid: Grupo Santillana de Ediciones S.A.

- Sarabia B. (1994). El aprendizaje y la enseñanza de las actitudes. En Coll et al. (Eds.), Los contenidos en la reforma: Enseñanza y aprendizaje de conceptos, procedimientos y actitudes (pp. 133-198). Madrid: Santillana de Ediciones S.A.

- Zapata, Z. (2006). Evaluación de contenidos y actividades de enseñanza de la Microbiología en textos escolares. Universidad Pedagógica Nacional - U.P.N. Licenciatura en Biología. 